


\title{
Desestabilización del principio de equivalencia como crisis intelectual en el pensamiento de Jean Baudrillard
}

\section{Destabilization of the principle of equivalence as an intellectual crisis in the thought of Jean Baudrillard}

\author{
Luis Alberto López Soto \\ Universidad de Sonora, México
}

Resumen: En el presente artículo se analiza un aspecto de la obra del sociólogo francés Jean Baudrillard, específicamente la noción de equivalencia, la cual funciona como principiodual válido en tres disciplinas: la lingüística, la economía y el psicoanálisis. Este principio es de naturaleza dual y se presupone como noción fundamental del paradigma moderno, de modo que estas disciplinas se hallan aquí representadas por tres autores fundamentales de la modernidad: Ferdinand de Saussure, Karl Marx y Sigmund Freud. Las equivalencias aquí estudiadas sonel signo lingüístico (significante-significado) en Saussure, la teoría del valor (valor de uso-valor de cambio) en Marx y la identidad del sujeto (o latente y lo patente) en Freud. Se hace una revisión del modo en que Baudrillard lee a estos tres autores con el objetivo de asociar aquel a la posmodernidad como crisis intelectual.

Palabras clave: Jean Baudrillard, equivalencia, posmodernidad, signo, crisis intelectual.

Abstract: This article will analyze an aspect of the work of the French sociologist Jean Baudrillard, specifically the notion of equivalence, which serves as a valid dual principle in three disciplines: Linguistics, Economics and Psychoanalysis. This principle is of a two-pronged nature, and is presupposed as a fundamental notion of the modern paradigm; these 
disciplines are therefore represented in this work by three fundamental authors of modernity: Ferdinand de Saussure, Karl Marx and Sigmund Freud. The equivalences studied here are the linguistic sign (signified and signifier) in Saussure; the labor theory of value (value in use and value in trade) in Marx, and the identity of the subject (or latent and patent) in Freud. A review is made of the way in which Baudrillard reads these three authors with the aim of associating his body of work with the idea of postmodernity as a crisis of the intellect.

Keywords: Jean Baudrillard, Equivalence, Postmodernity, Sign, Intellectual crisis.

\section{Equivalencia: una analogía matemática}

El término equivalencia connota una especie de matemática. Es común que los diccionarios filosóficos, sobre todo aquellos de filiación positivista como el de José Ferrater Mora, lo definan a partir de tal ciencia exacta. De ahí que en el presente artículo se entiende la equivalencia como una ecuación y, por ende, como un principio de medida para describir y explicar los fenómenos. Tomando esto como base, la noción de equivalencia se articula en otros ámbitos de saber para asumirse como científica y rigurosa. La equivalencia es, pues, un principio que se torna el asidero conceptual y la estrategia cognitiva a partir de la cual se establece una correspondencia, virtualmente sustentada en la realidad, entre términos pares que conllevan una dualidad, con el fin de explicar, en términos formales, lo existente. Así, el principio de equivalencia, al ser de inspiración matemática, busca configurar un enfoque de mensurabilidad para legitimar un discurso de saber.

En este tenor $-\mathrm{y}$ a propósito del origen matemático de la equivalencia- puede plantearse que, en virtud de una analogía con lo que se ha denominado como posmodernidad, hay actualmente una crisis de orden epistemológico que tiende a su vez sus implicaciones sociológicas y en el orden de las humanidades, toda vez que 
estas disciplinas han tendido -se sabe bien el debate entre estas dos culturas, léase el caso de Charles Pervy Snow, quien buscaba un diálogo en Las dos culturas y un segundo enfoque- a buscar moldes originados en las ciencias exactas. Así, en disciplinas como la lingüística, la economía y la psicología, la equivalencia -en tanto que sistema de pares- comporta una desarticulación, es decir, cierta desestabilización que representa un desafío a la forma dual que lo constituye, y se transfigura en pos de la ambivalencia o de un contínuum.

La naturaleza de esta crisis se halla, pues, relacionada con cierta imposibilidad para deducir una solución a partir de un sistema de pares, de modo que es el sujeto, maniatado para analizar, quien decide tal o cual solución, huelga decir, interpreta, completando así el fenómeno. Esto puede observarse en los famosos teoremas de Kurt Gödel (1906-1978), los cuales se exponen en un artículo de 1930 titulado "Sobre sentencias formalmente indecidibles de Principia Mathematica y Sistemas afines". En tal texto se argumenta que, dado un sistema formal (específicamente en un teorema), no es posible deducir una solución. Así, este fenómeno lógico-matemático como tal no resulta completo en sí mismo. En un plano menos técnico y más filosófico, Gödel plantea que las consideraciones de esta incompletud "no establecen limites de la capacidad de la razón humana, sino más bien de las posibilidades del puro formalismo en matemáticas" (Gödel, 1981: 197). Esta aportación, esta noción de incompletud de Gödel, sólo sería aplicable a las matemáticas en tanto que sistema formal objetivado, con lo que se intenta distinguir entre lo ecuacional y lo mental. Así pues, en el debate de si los procedimientos mecánicos pueden llevar más lejos que los procedimientos mentales, en una nota al pie agregada algunos años después al mencionado artículo, el matemático austriaco seńala que 
la mente, en su uso, no es estática, sino que está en constante desarrollo. Esto se ve, por ejemplo, considerando la serie infinita de los axiomas de infinitud cada vez más potentes en la teoría de conjuntos, cada uno de los cuales expresa una nueva idea o intuición (1981: 197).

Esta dinamicidad de la mente (que asociamos aquí a la razón y al pensamiento humano y, por extensión, a otras disciplinas como las mencionadas más arriba) no implica necesariamente una oposición a la mecanicidad, pues eso sería construir otra dualidad que escaparía a la desarticulación, que es la base de este trabajo. Sin embargo, tal distinción entre esos dos ámbitos (entre mente y mecanicidad), que permite explicar cómo es la naturaleza de la crisis aquí descrita y analizada, hunde sus raíces en el aspecto epistemológico y sociológico, es decir, más allá del aspecto exclusivamente matemático. Por lo tanto, esta dinamicidad no se trata nada más de una dialéctica hegeliana de la historia, revisitada ya por Marx, sino una lógica en la cual parecería harto complejo distinguir el ámbito del objeto y el ámbito del sujeto, en otros términos, entre la mecanicidad o "estaticidad" del objeto y la "dinamicidad" del sujeto. $\mathrm{Si}$, como dice Gödel, ciertos fenómenos lógico-matemáticos son indecidibles o indeductibles, se puede plantear, haciendo uso de cierta analogía para cuestionar la noción de las equivalencias, que los contenidos y las formas epistemológicas y sociológicas que aquí expondremos participan también de esa configuración.

\section{El caso de Jean Baudrillard}

Iniciada en El sistema de los objetos (1968), la obra del sociólogo y filósofo francés Jean Baudrillard es tanto una descripción como una interpretación del desarrollo social del siglo XXI occidental a la luz de ciertos principios de la lingüística, la economía y la psicología (el psicoanálisis). El resultado de esa descripción e interpreta- 
ción es una mixtura de sociología que echa mano de la semiología, o viceversa, de una semiología con cierta orientación sociológica. En suma, un pensamiento filosófico que, por su transdisciplinariedad, es de una amplitud inclasificable.

Sin embargo, para acotar su obra en un aspecto específico y pertinente para el caso que nos ocupa, cabe señalar que es de preponderancia, en las formulaciones conceptuales de Baudrillard, la noción de signo, sobre todo a partir de la publicación de Crítica de la economía politica del signo (1972). ${ }^{1}$ En este libro, el autor hace un análisis del desarrollo del capitalismo occidental, centrándose en la lógica dual del signo y tomando como base el signo lingüistico de Ferdinand de Saussure. Asimismo, realiza una crítica del materialismo histórico de Marx, quien concibe la mercancía como algo dual: valor de uso y valor de cambio. Revisa, además, la distinción freudiana entre el inconsciente y el consciente como una lógica de lo latente y lo patente. Así, en este trinomio de autores tenemos, pues, una configuración que asociamos a la equivalencia, toda vez que, a la luz de la lectura que Baudrillard realiza de estos autores, hallamos implicada una forma dual y ecuacional.

I) El primer uso que tiene el término equivalencia en Baudrillard está relacionado con el enfoque estructuralista de la lingüísti-

${ }^{1} \mathrm{Al}$ considerar los estudios sobre Jean Baudrillard, vemos que hay cierta unanimidad que formula la existencia de un "primer Baudrillard" (Kellner, 1989; Puig Peñalosa, 2000; Gane, 2000). Plantear que hay un "primer Baudrillard" supone, en términos lógicos, que hay un "posterior Baudrillard", es decir, una evolución autoral. Para efectos de este artículo, nos situaremos en ese "primer Baudrillard", el cual se halla todavía en un diálogo crítico con autores modernos. Cabe señalar que el punto culminante de esta etapa es la publicación de $E l$ intercambio simbólico y la muerte (1976), tiempo a partir del cual el "posterior Baudrillard" se aboca, por el tipo de categorías usadas, a un tipo de pensamiento que es tributario de la denominada posmodernidad. De algún modo, la crisis intelectual del caso de Jean Baudrillard se ve ejemplificada precisamente en la formulación de "primer Baudrillard" y un "posterior Baudrillard"; es decir, en un paso de la modernidad a la posmodernidad. 
ca de Ferdinand de Saussure. Si bien el término usado por Saussure en su mencionado Curso para definir el signo lingüístico es el de asociación (association), tal caso no deja de representar una suerte de equivalencia, toda vez que implica una dualidad que posibilita una resultante, es decir, una lógica de sumatoria, una lógica de ecuación. Para Saussure, el carácter de la lengua, o bien, de la relación entre las partes del signo lingüístico, es de naturaleza arbitraria, es decir, esta relación no responde a algún tipo de naturalidad o motivación intrínseca. Asimismo, Saussure ubica esta concepción del lenguaje en términos fundamentalmente sincrónicos (lo fijo y sistémico) en oposición a lo diacrónico (lo variable e histórico). La ecuación de esta equivalencia sería de la siguiente manera:

a) signo lingüístico: significante + significado.

II) El segundo uso que tiene el término equivalencia (équivalence) en la obra de Jean Baudrillard se halla en la mencionada obra, donde se refiere al valor de uso y el valor de cambio, conceptos economicistas analizados por Marx y que son la base de la cual parte Baudrillard para fundamentar su teoría sociológica del valorsigno. En tal obra, Baudrillard analiza la noción de mercancía, a la manera de Marx en el primer tomo de El capital, como la forma elemental del régimen de producción capitalista. La mercancía se compone, según Marx, por el trabajo (el tiempo cristalizado y empleado para su producción) y el valor de cambio (lo cuantitativo), lo cual sería una equivalencia de primer orden ("valor de cambio: trabajo + mercancía"). Esta equivalencia, a su vez, se subdivide en la siguiente:

b) mercancía $=$ valor de uso + valor de cambio.

III) El tercer uso que de dicho término hace Baudrillard es aquel relacionado con el psicoanálisis de Sigmund Freud. Hay en la obra de Freud, específicamente en El yo y el ello (1923) y en El malestar de la cultura (1930), una conceptualización sobre estos dos componentes ("el yo" y "el ello") donde se responde con una 
lógica dual para describir y explicarla constitución del sujeto y su comportamiento en el ámbito social y civilizatorio. A partir de esa dualidad Roland Barthes, en lo que él llama Mitologías recurre a una analogía epistemológica para afirmar que "el psiquismo [de Freud] es un espesor de equivalencias, un equivalete a” (111) y así referirse al entramado teórico con que Freud intenta describir y explicar el sujeto a partir de la relación entre la conducta patente o síntoma, el sentido de ésta (lo latente), con lo que la resultante es la correlación sumatoria de ambas. El esquema de esta equivalencia sería así:

c) identidad: conducta o síntoma (patente) + sentido (latente).

Así, resumiendo la interpolación que realiza Baudrillard de estos tres autores, se tiene que de la asociación entre significante y significado (Saussure), en el marco de la reglas del lenguaje y la comunicación que controlan el sentido; de la ambigüedad dada entre el valor de uso y el valor de cambio (Marx), y de ahí al valor/signo en el marco de la ley mercantil del valor y el capital; de la dualidad conducta patente/sentido latente (Freud), la visión de Baudrillard comporta una crítica cuyas implicaciones anotamos adelante.

En cuanto al ámbito de lo lingüístico, y considerando la primera equivalencia estudiada (signo lingüístico: significante-significado), se tiene que: a) la asociación arbitraria entre los dos elementos la ecuación evoluciona y es parte de una dinámica ambivalente. Estamos ante la tentativa de una relación significante-significado como la de una motivación (significante/significado), es decir, la de una relación natural, vivida socialmente como unívoca y que tiene su origen o correspondencia en cierta concepción "mágica" o premoderna. Bajo esta lógica, no habría tal equivalencia dada por una escisión abstracta y metafísica, sino una total articulación entre significante y significado como fusión inexorable y profunda entre forma y contenido. 
La consecuencia de esta crítica (o sea, la crítica de la crítica) que aquí se propone es que la razón moderna que pretende desarticular una relación de segundo orden (o sea, la relación lenguaje y pensamiento o lenguaje y realidad como analogía epistemológica de significante y significado) no repara en la idea y hecho de que todo el lenguaje es, más que un mero medio transparente y comunicativo, un punto de vista, ya pleno de sentido y significación, sobre la realidad de la cual sólo se puede saber precisamente a partir de la instancia del lenguaje. De este modo, quedaría deconstruida la relación arbitraria entre significante y significado para configurarse "mágicamente" como una motivación, la cual es, en realidad, una ambivalencia, la paradoja de que los elementos del signo lingüístico funcionan, más que una dualidad como destino estructural, como una mera reducción tanto sociológica como fenomenológica.

Asimismo, la lectura de Baudrillard aquí propuesta es que, en suma, la equivalencia moderna tendría en la posmodernidad una crítica premoderna que postula una ambivalencia lingüística (extendida a su vez a lo sociológico y epistemológico), la cual funcionaría como una categoría más dinámica frente a la cristalización de la dualidad hallada en Saussure. En definitiva, la posmodernidad en Baudrillard se halla asociada a una premodernidad, fenómeno teórico que aquí se interpretamos por demás ontológicamente paradójico y ambivalente, toda vez que implica volver a una concepción del signo lingüístico como un elemento cuyos componentes no son arbitrarios, sino motivados; concepción eminentemente premoderna.

En cuanto al ámbito del valor económico, y considerando las segundas equivalencias estudiadas (mercancía: valor de uso y valor de cambio), se tiene que: $b$ ) las nociones de valor de uso y valor de cambio como dualidad de la ley mercantil del valor (léase economistas clásicos) que, en Marx (léase El capital), pasa por una crítica 
económica y filosófica al ser incorporado el elemento humano (la fuerza de trabajo) a la lógica de la mercancía y del capital, es sometida por Baudrillard a una crítica radical. La deconstrucción del valor de uso se halla en la idea de que éste no es sino una emanación sobredeterminada del valor de cambio, una imposición de lo cuantitativo sobre lo cualitativo. Esta dinámica incierta se halla a la vez asociada a la función del trabajo humano (vivo) y en pos del trabajo de las máquinas (muerto) como principio y elemento sine qua non de la ecuación del valor y del valor de cambio. En Baudrillard, el carácter equivalente de los elementos de esta dualidad termina no sólo por pasar a una ambivalencia -donde ya es indeterminable dónde empieza lo cuantitativo y dónde inicia lo cualitativo-, e incluso el carácter del valor no sólo pasa a sintetizarse dialéctica y hegelianamente en la noción de valor-signo, sino que pasa a una etapa fractal del valor, a una desestructuración total del valor

La consecuencia de esta crítica radical es, a juicio de lo aquí propuesto, que tal desestructuración del valor es, en realidad, la de una abstracción del valor donde los elementos implicados (trabajo, mercancía) son ya una mera función derivativa. O bien, esta abstracción está ya toda ella implicada en la concretización, es decir, en la ilusión de un valor de uso concreto, donde el trabajo (la acción humana) queda así elidida, descentrada. Es, pues, una visión de lo abstracto a lo concreto. Contrariamente a esto, el método de investigación marxista, distinto al de exposición, es una lógica racional y empírica que va de lo real concreto y de ahí a lo abstracto y viceversa, siguiendo así una dialéctica que deduce el modo en que los fenómenos se producen en su dimensión material más precisa. En la visión asistemática de Baudrillard, el método de investigación y el de exposición están tácitamente fundidos. Si Marx se niega a partir del método poblacional (o sea, de una generalidad inasible y, por lo tanto, imprecisa), Baudrillard parte más bien de la noción de masa, noción tan inasible como la de población, la 
cual no sólo es abstraída, sino abstracta en sí misma. Es, pues, esta perspectiva de la masa como algo central en las sociedades postindustriales, pero no desde la mirada del intelectual que se convierte en conciencia, a la manera del marxismo revolucionario o del humanismo liberal, sino del intelectual abstraído, ajeno e indiferente a todo proyecto de lucha y liberación. Esta sería la diferencia conceptual entre la mirada moderna y la mirada baudrillardiana acerca de la masa como fenómeno y como tema semiótico y sociológico.

Así pues, esta fusión de métodos y vuelta a la abstracción en el orden de lo epistemológico tiene, a su vez, una correspondencia con otra abstracción en el orden del contenido analizado (lo sociológico) en la teoría de Baudrillard: la idea de que el capital ha producido no sólo mercancías, sino que el capital ha tendido a reproducir la lógica de las relaciones sociales con el fin de perpetuarse. No sólo el objeto concreto, sino la idea, lo abstracto del objeto y del sujeto. El capital no sólo como productor de mercancías para ser consumidas, e incluso no sólo como productor de sujetos consumidores, sino como una instancia abrumadoramente reproductiva de la lógica social del capital en todos los ámbitos y ya en dimensiones transnacionales. He ahí una configuración avanzada que, desde la equivalencia, se replantea no sólo los contenidos sociológicos a describir sino la forma epistemológicas de razonar.

En cuanto ámbito de lo psicológico y/o psicoanalítico, y considerando la tercera equivalencia estudiada, se tiene que: $c$ ) la lógica dual de lo patente y lo latente en Freud ha fungido como el modo a partir del cual se razona el fenómeno de la psique y cuyo propósito es traducir a términos racionales y conscientes las dinámicas del inconsciente, la libido y demás pulsiones, presumiblemente innatas. Así, el psicoanálisis se enmarca en una visión que divide al sujeto en dos, enfoque que, sociológicamente y en términos del mismo Freud, se corresponde con un principio de placer y un principio de realidad. El ego o la identidad del sujeto sería la resultante de 
estos dos elementos, la síntesis de esta articulación. Sin embargo, la lectura baudrillardiana toma la lógica de lo inconsciente para aplicarla en el fenómeno amplio del consumo. El consumo tendría una lógica inconsciente, es decir, un orden preestablecido pero en ningún modo innato, sino dado por el sistema actual como un mecanismo de asimilación a partir de la apelación mercantil y libidinal de generación de deseo y, por ende, de placer. El sujeto se convierte o se constituye como tal en tanto que es consumidor. Para Herbert Marcuse, este es un proceso de desublimación represiva, toda vez que el sujeto no consigue liberarse de las fuerzas externas (Marcuse, 1993: 102). Su ello, su libido, en suma, todas las instancias latentes terminan por ser neutralizadas o reprimidas filosófica y, sobre todo, políticamente. En cambio, a partir del concepto de desublimación dirigida de Baudrillard, se intenta, más que nada, describir un proceso que no podría ser represivo sino meramente sistémico, al descreer de una esencia, una ontología del sujeto que podría ser liberada. Con base en esto, la enajenación del sujeto productor y, más tarde, del sujeto consumidor, quedaría entredicha.

La consecuencia de esta crítica es que hay un desmonte de cualquier posibilidad de hablar de un equilibrio entre lo patente y lo latente, pues aquel se impondría inevitablemente sobre éste, y, en tal caso, como concepto sustantivo, lo latente sólo podría funcionar como una mera ficción explicativa. La estimulación del medio, es decir, el consumo como fuerza histórico-social y como modo de reorganización lógica del capital, construye en sí mismo toda una pulsión. No habría, pues, sino racionalización y universalización de una instancia histórica. Si lo patente es la conducta medida y observable, la paradoja aquí ubicada es que lo patente sea, transparentemente en una especie de tautología epistemológica, lo único real verificable. 


\section{Posmodernidad y actitud hermenéutica}

Cabe hacer una conceptualización al respecto de la relación de la modernidad, posmodernidad y el pensamiento baudrillardiano. Según Francois Lyotard, en su ya conocido ensayo "La condición posmoderna. Informe sobre el saber" (1979), el término posmodernidad "designa el estado de la cultura después de las transformaciones que han afectado a las reglas de juego de la ciencia, de la literatura y de las artes a partir del siglo XIX" (1979: 4). Lyotard parte de la base de que toda ciencia -amén de buscar lo útil y lo verdadero- se opone a un relato. Ante esto, la ciencia busca, a su vez, corroborar sus reglas de juego. Asimismo, la filosofía, en tanto que ciencia, busca también "su propio estatuto de legitimación" (4). De este modo, un metarrelato resulta cuando un discurso de saber (la filosofía, en este caso) que apela a un relato más amplio -sea éste el de una "dialéctica del Espíritu, la hermenéutica del sentido, la emancipación del sujeto razonante o trabajador" (4)-. Este procedimiento conceptual no hace sino presuponer su principio en una dinámica consensuada entre los hablantes ("emisor y destinatario"), estableciéndose con esto los valores de verdad y los criterios de verificación.

Para Lyotard, la crítica que evidencia tales metarrelatos es de carácter posmoderno, con lo que intenta asociar la modernidad a una racionalidad newtoniana, centrada en una filosofía metafísica y en toda una institución universitaria sustentadora de tales perspectivas. La crítica posmoderna se muestra, pues, escéptica de estas tendencias que se configuran como metarrelatos para dar pie a la instancia del lenguaje, de los juegos del lenguaje; es decir, elementos epistemológicos inestables.

Así, haciendo una extrapolación, las equivalencias modernas de los autores cuestionados por Baudrillard son muestra de un metarrelato, con lo que, a grandes rasgos, se puede definir el pen- 
samiento de Baudrillard como posmoderno. En lo específico, el pensamiento -que podríamos definir genéricamente como posmoderno- de Jean Baudrillard participa filosóficamente de lo que, a grandes rasgos, describiríamos como una versión extrema de la hermenéutica. De hecho, gran parte del pensamiento posmoderno puede considerarse como perteneciente a este rubro. En términos generales, se ha entendido como hermenéutico a un tipo de pensamiento que se aboca a la interpretación, más que al análisis, es decir, un enfoque y actitud disciplinares que, agotando las posibilidades de todo desciframiento o de toda facultad investigativa, da pie para que se termine por realizar una reducción, una interpretación. Nacida en la interpretación de textos sagrados, y de algún modo interrelacionada con la semiótica en tanto que preocupada por atribuirle sentido a un signo, la hermenéutica aspiraría a interpretar todo fenómeno, la realidad misma, ya no sólo como un texto sagrado o secular, sino como una totalidad asible que incluye, por supuesto, todo tipo de textos.

En el caso de la obra general de Baudrillard, y en lo particular sobre las equivalencias y cuya desestabilización hemos deducido definiéndola como una crisis, el pensamiento baudrillardiano descrito, analizado y problematizado en este trabajo participa de un modo hermenéutico de saber, en la medida que parecería negarse al análisis empírico de datos.

De algún modo, en El conflicto de las interpretaciones (1969) de Paul Ricoeur - uno de los representantes de la hermenéutica-, se halla, a cierto nivel, esta crisis que hemos venido exponiendo. Como bien lo dice el título, es decir, como parte de un conflicto, una pugna crítica, esta obra de Ricoeur pone de relieve las interpretaciones en el ámbito de la comprensión de la existencia. Ora en signos, ora en símbolos, la existencia, según esta lectura de Ricoeur, conlleva un sentido, o bien, sentidos susceptibles de ser descifrados. 
En su versión contraria, se halla lo que se conoce como filosofía analítica, de herencia positivista, y cuyo principal representante sería Bertrand Russell. En este tipo de pensamiento el margen de interpretación es mínimo, pues, a decir de esta tendencia, el objeto de estudio como tal sólo puede ser conocido empíricamente por el sujeto a partir del método científico, basado este último en el de las ciencias naturales. La racionalidad moderna es tributaria en gran medida de esta concepción y, por lo tanto, vive en cierta deuda con las ciencias naturales.

Hemos ya planteado cómo Baudrillard lee a Marx, Saussure y Freud. Éstos cumplen cierta función de fundadores de discursividad, la cual es una noción que, a decir de Foucault (6: 1998), consiste en la construcción de una disciplina cuyo potencial y alcances escapan a las aportaciones de su fundador. Así, como se ha observado, el marxismo escapa al mismo Marx; la lingüística estructuralista, a Saussure, y el psicoanálisis, a Freud. De este modo, la tríada de estos discursos pertenecen a un orden social, el de la modernidad, y hay en ellos cierta presunción científica, es decir, de ciencia a la manera moderna.

Ahora bien, si bien no se pueden equiparar los elementos concretos de la filosofía analítica con las nociones aquí estudiadas, e interpretadas como muestra de un desestabilización posmoderna en el pensamiento de Baudrillard, la racionalidad moderna implicada en Marx, Saussure y Freud estaría por lo menos más cerca de la visión analítica que de la interpretativa. En otros términos, la intención de estos autores es la de hacer una contribución a la ciencia con métodos tan presumiblemente válidos como los de la ciencias empíricas, y no tanto una labor donde el sujeto interpreta libremente tal o cual fenómeno, sea el del lenguaje, la economía o el psiquismo. No es sólo un discurso de saber que intenta deducir, inducir, establecer analogías, en fin, todo ese tipo de razonamientos, sino, en el mundo occidental, el discurso de saber por defini- 
ción. Esa es, pues, el legitimación a través de la cual la racionalidad moderna pretende constituir su carácter científico.

Al pensar estos dos enfoques de pensamiento (el de la filosofía analítica y el de los fundadores de discursividad: Saussure, Marx y Freud) en relación a la hermenéutica, puede sugerirse un esquema en el cual se dividen gradualmente todas estas disciplinas:

Ciencias naturales.........Filosofía analítica.........Hermenéutica

Lingüística estructuralista (significado-significante)

Marxismo (valor de uso-calor de cambio)

Psicoanálisis (lo latente-lo patente)

El análisis................................La interpretación

Lo empírico-factual........................Lo mental-racional

El objeto.......................................

UNIVOCIDAD......Hermenéutica anal......EQUIVOCIDAD

En este esquema, la obra baudrillardiana funge de elemento crítico que se ubica gradualmente en la disciplina hermenéutica, toda vez que se ocupa no sólo de una descripción y un análisis semióticos de las sociedades occidentales en el capitalismo avanzado, sino de una interpretación sumaria de los fenómenos. (A propósito de esto, puede decirse que la hermenéutica ha tenido la connotación de una menor cientificidad, siempre y cuando se defina a ciencia como mera transferencia de datos que dan cuenta ineludiblemente de lo objetivo previa verificación, casi sin participación crítica del sujeto.)

Para matizar esta serie de ideas, estableceríamos que la de Baudrillard es más bien una actitud hermenéutica, no tanto una filiación a la hermenéutica como modo de saber. Una vez que ha analizado sistémicamente diversos aspectos de la economía, del lenguaje, de la psicología, Baudrillard adopta una visión hermenéutica. Así, considerando la gradualidad que se presenta en el cuadro anterior, puede observarse que Baudrillard intenta partir de un análisis para abocarse a la interpretación, de lo uní- 
voco a lo equívoco, es decir, de lo sistémico a lo singular, en suma, de una modernidad crítica a una posmodernidad acrítica.

Así, por ejemplo, al hablar del carácter preponderante de la mercancía en Marx y del signo en Baudrillard, este, en su interpretación, termina invirtiendo el orden:

mercancía (valor de uso y valor de cambio) $\rightarrow$ signo

signo $\rightarrow$ mercancía

Así también en el esquema saussuriano del significante-significado, y a propósito del debate de Baudrillard con Benveniste sobre la intercalación del referente expuesto en el capítulo tres, puede plantearte otra inversión:

referente (significante y significado) $\rightarrow$ signo

signo $\rightarrow$ referente

Así también en la lógica dual de lo patente y lo latente, del comportamiento y el síntoma en Freud, es decir, conducta patente/ sentido latente, se observa:

conducta (lo patente y lo latente) $\rightarrow$ signo

signo $\rightarrow$ conducta

De este modo, se ha pasado del hecho concreto de la mercancía al hecho abstracto del signo, del hecho concreto del referente a la idea abstracta del signo y del hecho concreto del comportamiento al signo como síntoma. En estas inversiones observamos, pues, una estrategia hermenéutica de los conceptos, de manera que la mercancía, el referente y la conducta como tales (pertenecientes al ámbito de lo factual, lo analizable materialmente, lo empírico, lo objetual) quedan relegadas a un segundo plano, y el signo, que no es sino una mera virtualidad, resulta preponderante.

Esta preponderancia del signo redunda, según nuestro punto de vista, en una visión hermenéutica toda vez que implica que el signo como tal, como hecho objetivo e inmanente, no nace sino de la mirada interpretativa en un sentido epistemológico y sociológico, y no sólo psicológicamente como planteaba Saussure en el Curso de lingüistica general. Así pues, no hay signo sin la posibilidad 
de una instancia de interpretación. La preponderancia del signo da pie, a su vez, a la lógica hermeneutizante.

En la obra de Charles Sanders Peirce, hallamos la noción de interpretante (1987: 245), la cual figura como un tercer elemento que media entre el objeto (el referente) y el signo ("representamen”). Ahora bien, la relación entre este interpretante o intérprete y la hermenéutica posmoderna estaría articulada por el signo. En el dominio del signo no hay más que este y el intérprete. En un sentido radical, el mundo en su totalidad sería una serie de signos. Que Baudrillard invierta este orden y pugne por la precesión del signo es, en sí mismo, un indicio de un paradigma distinto al de la modernidad, pues socava la relación entre sujeto y objeto: el objeto es signo y el sujeto es el intérprete, pero el objeto como tal precede al sujeto mismo. Este nuevo paradigma consiste, pues, en una actitud interpretativa que, al decir de Gadamer,

requirió a la larga apartar la conciencia histórica de la presión del ideal objetivista de la ciencia moderna y desarrollarla como una conciencia hermenéutica que permitiera al mismo tiempo distancia y penetración. La historia no es entonces tanto objeto de una ciencia, sino más bien la ciencia es una parte de nuestro talento (Gadamer, 1990: 155).

Hallamos que esa "distancia y penetración" comporta, en primera instancia, una reconceptualización de las categorías de "la ciencia”, acotadas aquí en las consabidas equivalencias. Esta reconceptualización implica, además, una reducción total de lo fenoménico al signo. $\mathrm{O}$ bien, la reducción fenomenológica se vuelve una decodificación e interpretación del signo. Esa actitud interpretativa se halla imbricada, para nuestro propósito, en la problemática del derrumbe de las equivalencias en Baudrillard, la cual se ha entendido como la de una crisis, es decir, un cambio paradigmático, pues inicia suponiendo un replanteamiento total de ciertos 
presupuestos hasta llegar a cierto límite de análisis. Así, se deja de analizar para sólo interpretar.

Sin embargo, tal condición interpretante no puede ser sino la de una crisis y una paradoja, toda vez que, en el caso de Baudrillard, estamos frente a un pensamiento que, como se ha analizado, suscita un escepticismo extremo y radical:

No existe un equivalente para el mundo. En eso consiste precisamente su definición, o su indefinición. Sin equivalente, no hay doble ni representación, ni espejo. Cualquier espejo seguiría formando parte del mundo. No hay sitio para el mundo y para su doble al mismo tiempo. Por lo tanto, no hay verificación posible del mundo, razón por la cual la "realidad" es una impostura (Baudrillard, 1999: 5).

Estas afirmaciones, ya en las vísperas del siglo XXI, deben leerse como muestra de una actitud hermenéutica que, participante de la posmodernidad, se vuelca en un nihilismo posterior al análisis tanto empírico como racional. Cuando Baudrillard afirma que "sin equivalente, no hay doble ni representación”, está leyendo hermenéuticamente: o sea, interpreta la totalidad presumiblemente asible tan sólo para negarla e intentar "refutarla".

Asimismo, es una reducción fenomenológica toda vez que hay en Baudrillard el planteamiento no sólo de una insuficiencia de tal o cual equivalencia (sea en Saussure, en Marx o en Freud), sino la noción del mundo total como carente de equivalente alguno, un mundo (una filosofía, una sociología y una antropología), para usar una metáfora gramatical, sin función predicativa. Y es este mundo conceptual de las dualidades el que funciona como la ilusión de la totalidad del ser.

De este modo, en tanto que elemento constitutivo de la posmodernidad, esta crisis pasa, pues, por una ambivalencia. Esta crisis puede definirse tanto como la agudización de la crítica como una 
especie de regresión, de un tipo de acrítica. Aún más, si se puede radicalizar esta perspectiva, se diría que la sola ambivalencia como tal no explicaría las condiciones actuales (al derrumbarse las equivalencias) del mundo moderno occidental. Hay entre la equivalencia y la ambivalencia, además, una hipótesis alternativa: la de un continuum. Si la equivalencia admite una dualidad inherente que funciona como una especie de traducción cualitativa (la lógica de "lo que es aquí, es allá; como es aquí, es allá"), y si la ambivalencia propugna por una contradicción de orden paradójico (la lógica de "esto es y no es como aquello"), el continuum que como alternativa teórica se observa aquí parecería dar pie a un orden más bien relativo. Es decir, el continuum representa el dominio total de lo cuantitativo sobre lo cualitativo y, por lo tanto, de un aspecto más de la desestabilización que hemos postulado, toda vez que implica sobre todo plantear una gradualidad incierta frente a la equivalencia y a la ambivalencia. Para usar una metáfora proveniente de la astronomía, el continuum sería una especie de agujero negro entre la equivalencia y la ambivalencia.

Tal continuum puede observarse, al referirse Baudrillard a la mercancía en Crítica de la economía política del signo, en "una lógica funcional del valor de uso; una lógica económica del valor de cambio; una lógica del cambio simbólico; una lógica del valor/ signo", donde "La primera es una lógica de las operaciones prácticas. La segunda es una lógica de la equivalencia. La tercera es una lógica de la ambivalencia. La cuarta es una lógica dela diferencia" (Baudrillard, 1974: 56-57). A manera de diagnóstico, nuestra lectura es que este continuum relativiza todas estas formas y todas esas lógicas, es decir, tanto la practicidad como la equivalencia, la ambivalencia y la diferencia, son ya un amplio espectro que lineal y cuantitativamente van complejizándose, de modo que el intérprete, el hermeneuta, el autor posmoderno selecciona -de acuerdo a su particular criterio en el amplio espectro y partiendo de la pri- 
mera hasta la cuarta lógica- cuál grado de relación se referirá. Así, la selección es ya un punto de vista comprometido de origen con tal o cual fenómenos problematizado, llámese mercancía, objetos, deseos, etcétera.

Cabe hacer una articulación entre un tipo de hermenéutica y la crisis aquí aludida, con el fin de entender de qué modo el pensamiento baudrillardiano participa de un espectro disciplinario más amplio en torno a las equivalencias. Una versión más específica de la hermenéutica atiende y se refiere a esta crisis de desestabilización como un problema intelectual que puede ser subsanado a partir de lo que se denominaría hermenéutica analógica-icónica. Es, pues, la propuesta del filósofo mexicano Mauricio Beuchot, para quien las condiciones actuales de "caída del sentido, de falta de símbolos que señalen el camino [...] en un tiempo brumoso, nada halagüeño, de crisis. [...] Crisis de la razón. Crisis de sentido y de valores" (Beuchot, 2013: 21) pueden ser entendidas, en primera instancia, a partir de la distinción entre univocidad y equivocidad. Univocidad ahí donde la filosofía moderna, analítica, positivista, precisa de conceptos y categorías claras para aprehender la realidad; equivocidad ahí donde el nihilismo contribuye a desdibujar las categorías en una disolución de la realidad. Modernidad: univocidad, unión e integración; posmodernidad: equivocidad, separación y difuminación. La primera conduce al literalismo, a la rigidez, a la exactitud en una distinción tajante entre sujeto y objeto y demás asociaciones (o, diríamos, disociaciones) epistemológicas; la segunda, a la metáfora, a la relajación total de los criterios, a un relativismo extremo.

Con base en esto último, podemos decir que estamos frente a una nueva dualidad, de modo que, para Beuchot, se hace necesario integrar estas dos posibilidades, es decir, recurrir a cierta proporcionalidad, cierta visión hermenéutica que funja de mediadora para no ceder frente a los extremos y recuperar así el sentido. 
Esta dualidad se halla profundamente relacionada con la consabida problemática del signo en general y con las configuraciones epistemológicas y sociales (lingüística saussuriana, economía marxista, psicología freudiana) en particular. Asimismo, como contraparte de la dualidad, hallamos la función de la lógica del símbolo, pues este pertenece no a lo dual, sino a lo unitivo, a la motivación. El símbolo es el tipo de signo cuya asociación es casi natural. Según Beuchot, el símbolo, la lógica simbólica y, por ende, el modo de organización social (su ontología y su axiología) que de aquellos emanan, son aún vigentes, si bien de un modo bastante acotado. Como se ha visto, Baudrillard sostiene lo contrario, y tal pensamiento entraría, según las categorías de Beuchot, en el equivocismo que, en tanto que tal, no construye el sentido de los fenómenos, ya de por sí obnubilantes, sino que desfigura toda noción de orden y proporción. Así, en la gradualidad que va desde la practicidad, a la equivalencia, y de ahí a la ambivalencia hasta llegar a la diferencia, parecería predominar la diferencia, es decir, el relativismo.

Ante tal disyuntiva, sostenemos que, en lo que respecta a las equivalencias estudiadas como emanación del signo y a la arbitrariedad dual de este, y a la ambivalencia como parte inherente del símbolo, la ecuación (para usar un término matemático) es provisionalmente indecidible. Es decir, entre la postura de Beuchot y de Baudrillard se conformarían cierta indecidibilidad. Conforman, pues, una antinomia.

Para aplicar el esquema de Beuchot y retomar nuestra propuesta de análisis en cuanto a las funciones disciplinares de las ciencias aquí aludidas en este apartado, se puede plantear el siguiente esquema: 
SIGNO

SÍMBOLO

EQUIVALENCIA

AMBIVALENCIA

Ciencias naturales.

.Filosofía analítica

Hermenéutica

Lingüística estructuralista (significado-significante)

Marxismo (valor de uso-calor de cambio)

Psicoanálisis (lo latente-lo patente)

El análisis

La interpretación

Lo empírico-factual...................................... Lo mental-racional

El objeto...........................................

UNIVOCIDAD...Hermenéutica analógica....EQUIVOCIDAD

El área punteada representa ese continuum que funge de alternativa a la dualidad sociológica y epistemológica de las equivalencias, una vez que la lógica del símbolo (unitiva, no dual) ha sido, valga la expresión, "descontinuada", o por lo menos, acotada. Este continuum es, asimismo, la alegoría de la desestabilización en el orden de lo intelectual que aquí ubicamos en el desarrollo del pensamiento de Jean Baudrillard, un pensamiento tensionado entre la semiótica como disciplina de análisis y la hermenéutica como disciplina de reducción fenomenológica.

\section{Conclusiones}

A lo largo de este trabajo se ha planteado cómo es que la filosofía y la sociología baudrillardianas, en específico el tema del principio de las equivalencias, comportan una lógica semiótica, toda vez que los fenómenos tanto conceptuales como sociales se presentan como propios de un lenguaje, es decir, de una serie de signos. $\mathrm{O}$ viceversa, cómo es que tal semiótica tiene, por el contenido mismo de los fenómenos analizados, fuertes implicaciones directas en la sociología y en la filosofía. Tal es la perspectiva aquí tratada.

Ahora bien, trayendo a colación la mecanicidad de los sistemas objetivados por la matemática en el teorema de Gödel aludido, en 
las ciencias empíricas, y, por ende, en las ecuaciones de las equivalencias como formas duales aquí estudiadas -léase, mercancía = valor de uso + valor de cambio; signo lingüístico = significante + significado; identidad $=$ conducta $($ patente $)+$ sentido (latente) - , hallamos que tal incompletud objetivada sería la coartada a partir del cual se construye el pensamiento, es decir, toda la constitución del sujeto como intérprete, como hermeneuta (acotado aquí en las formulaciones de Baudrillard). Es, pues, una coartada del dualismo que consiste en dividir y reducir, sea al signo, al símbolo o al contínuum, en pos de un sentido explicativo de los fenómenos. Ante la imposibilidad de pasar, en una sumatoria, de un lado de la ecuación hacia el lado en una sumatoria racional (o sea, en la transición), se suspende el análisis y termina por hacerse una interpretación. Es ahí donde el mismo pensamiento de Baudrillard queda varado por su crítica, la cual, como sostenemos, evidencia una crisis, una crisis basada en la indecibilidad y en la indemostrabilidad.

Huelga decir la paradoja que consiste en demostrar la indecibilidad, es decir, en demostrar la propia indemostrabilidad. Pero tal es la condición de lo aquí descrito y analizado. Así, como en los teoremas aludidos, en este sistema formal (el de las equivalencias) esta crisis que hemos expuesto y analizado es tan radical que es sintomática de un pensamiento que secreta su propia insuficiencia, toda vez que se agota en el devenir histórico, en la interpretación coyuntural de una etapa del pensamiento, es decir, en lo que aquí hemos tratado como sociológico y epistemológico.

Sea la de la economía política, la del significante, la de la identidad del sujeto, la modernidad en estos saberes es la de una crítica, mas es, sin embargo, una crítica organizadora, convergente, dispuesta en cierta manera a contribuir al pensamiento racional occidental. En el caso de Baudrillard, no sólo es insuficiencia, sino un intento de refutabilidad del concepto mismo de realidad como forma epistemológica, más allá de su contenido de naturaleza social. 
Así pues, tenemos que de la noción matemática de equivalencia hemos pasado a la noción matemática de indecidibilidad: de una forma por demás estable, pasamos a la noción incierta e inestable por naturaleza de la indecidibilidad. Y es frente a este fenómeno de indecibilidad y de incompletud donde el sujeto intelectual se ve impelido a la interpretación. Asumida como lo fenoménico y como presupuestos conceptuales, la realidad objetiva comporta cierta indecidibilidad e incompletud, en oposición al sujeto, quien "decide", esto es, completa la ecuación y reduce, maniatado, no obstante, por la densidad de lo real en la indagación del sentido. Así, en la filosofía analítica, la racionalidad moderna alcanza sus límites y la hermenéutica entra a escena como un mecanismo de complementación. La modernidad como un límite y la posmodernidad como un estado varado de configuraciones intelectuales donde se accede a estrategias de saberes y en el cual lo lógico-epistemológico no escapa a lo social. Para Baudrillard, esta relación sujeto-objeto se ha trastrocado, diluido, pues, para este, el sujeto no decide. Y el sujeto no está, en ningún modo, un proceso dialéctico. No así para Beuchot, pues de ser así, razona este, la realidad sería incognoscible, y todo discurso sería un mero acto absurdo de solipsismo.

En nuestro caso, los fenómenos se han acotado al ámbito de los signos, los cuales han perdido ya la unicidad del símbolo (lo ambivalente, lo motivado, lo total) como encarnación de lo concreto para desplegarse en una configuración dual (lo lingüístico en Saussure, lo económico en Marx, lo psíquico en Freud) como cúspide de la abstracción (lo equivalente, la arbitrariedad, la escisión). Tal es la naturaleza de la crisis epistemológica que ha enfrentado el pensamiento occidental en lo que va de la modernidad a la posmodernidad. Es una crisis que, frente a la complejidad de los fenómenos, tiende a constituir un sujeto débil (como diría otro hermeneuta posmoderno como es Gianni Vattimo) quien deja de 
analizar y se orienta a interpretar y así otorgar sentido a sus asociaciones conceptuales en la búsqueda de explicación del movimiento de la realidad.

A partir de estas dos posiciones (la de Baudrillard y la de Beuchot), deducimos que hay en el ambiente actual un afán de refutabilidad de lo real y de la esencia de lo social que consiste en un cuestionamiento de la mensurabilidad dual (aspecto epistemológico) para arrojar luz sobre el desarrollo de la civilización occidental del siglo XX (aspecto sociológico). Asimismo, hay una inextricable relación entre sujeto (interpretante) y los fenómenos como objeto de estudio. La implicación de esto es, además, que la posición de Baudrillard acerca del cuestionamiento de las equivalencias se muestra provisionalmente, al igual que los teoremas de Gödel, indecidible.

Después de todo esto, la denominada crisis intelectual puede definirse como un punto de inflexión donde se intercalan las disciplinadas aquí implicadas (semiótica y hermenéutica). Esta disciplinas se asociadas en la relación, tanto de continuidad como de ruptura, entre la modernidad y la posmodernidad. Y en el marco de estas disciplinas se toma como corpus algunos aspectos de la obra de Jean Baudrillard utilizados para desembocar en el tema de las equivalencias (significante-significado, valor de uso-valor de cambio, lo latente-lo patente).

\section{Bibliografía}

Barthes, Roland, 1999, Mitologías, 22da. Edición, Héctor Scmucler (trad.), Siglo XXI editores, Madrid.

Baudrillard, Jean, 1999, El intercambio imposible, Alicia Martorell (trad.), Cátedra, Colección Teorema, Madrid.

Baudrillard, Jean, 1974, Crítica de la economía política del signo, Aurelio Garzón del Camino(trad.), Siglo XXI, México. 
Beuchot, Mauricio, 2015, Tratado de hermenéutica analógica, 5ta. edición, UNAM, Cuadernos del Seminario de Hermenéutica, México.

, 2013, Las caras del símbolo: el ícono y el símbolo, BUAP, Colección La Abeja de Perséfone, Puebla, México.

Broker, Alfred, 1988, "El Marx de Baudrillard", en Modernidad y posmodernidad, Alianza, Madrid, pp. 293-319.

Butler, R., 1999, Jean Baudrillard: The Defense of the Real, Sage, London.

Ferrater, José, Diccionario filosófico, t. I, 5ta. edición, Sudamericana, Buenos Aires, 1964.

Foucault, Michel, 2010, ¿Qué es un autor?, Silvio Mattoni (trad.), Literales, Colección Cuadernos de plata, Córdoba.

Freud, Sigmund, 1993a, "Introducción del narcisismo", Obras completas, t. XIV, José Luis Etcheverry (trad.), Amorrortu Editores, Buenos Aires, pp. 71-98.

, 1993b, "El yo y el ello y otras obras", Obras completas, t. XIX, Buenos Aires, Amorrortu Editores, 1993, pp. 334.

Gauthier, Alain, 2008, Baudrillard Unepenséesingulière, Lignes, París.

Gödel, Kurt, 1981, Obras completas, José Mosterín (intro., trad.), Alianza, Madrid.

Gadamer, Hans-Georg, 1990, La herencia de Europa. Ensayos, Pilar Giralt (trad.), Península, Barcelona.

Lyotard, Francois, 1993, La condición posmoderna, Mariano Antolín Rato (trad.), Planeta/Agostini, Barcelona.

Marcuse, Herbert, 1993, El hombre unidimensional, Antonio Elorza (trad.), Planeta, México.

Marx, Karl, 1974, La ideología alemana, 5ta. Edición, Wenceslao Roces (trad.), Ediciones Pueblos Unidos, Buenos Aires. 

xico.

, 2010,El capital, t. I, Pedro Scaron (trad.), Siglo XXI, Mé, 1989, Contribución a la crítica de la economía politica, 9na. Edición, Jorge Tula et al (trad.), Siglo XXI Editores, México.

Pervy, Charles, 1988, Las dos culturas, Horacio Pons (trad.), Ediciones Nueva Visión, Buenos Aires.

Pierce Charles Sanders, 1987, Obra lógico-semiótica, Ramón Alcalde y Mauricio Prelooker (trad.), Taurus, Madrid.

Puig Peñalosa, Xavier, 2000, La crisis de la representación en la era posmoderna: El caso de Jean Baudrillard, Abya-Yala, Quito, Ecuador.

Ricoeur, Paul, 2003, El conflicto de las interpretaciones, Alejandrina Falcón (trad.), FCE, México.

, 1990, Freud: una interpretación de la cultura, 8va. Edición, Suárez et al (trad.), Siglo XXI Editores, México.

Russell, Bertrand, 1966, Ensayossobrelógica y conocimiento, Javier Murgueza (trad.), Taurus, Madrid.

Recibido: 1 de marzo de 2017 Aceptado: 11 de septiembre de 2017 